\title{
Fosfito de potássio no controle do míldio da videira e características físico-químicas de uvas Merlot
}

\author{
Vanessa Foresti Pereira(1) ${ }^{(1)}$ Mário Lúcio Vilela de Resende( ${ }^{(1)}$, Pedro Martins Ribeiro Júnior ${ }^{(1)}$, \\ Murillo de Albuquerque Regina( ${ }^{(2)}$, Renata Vieira da Mota $^{(2)}$ e Luiz Rodolpho Rodrigues Vitorino ${ }^{(1)}$ \\ (1)Universidade Federal de Lavras, Departamento de Fitopatologia, Campus Ufla, Caixa Postal 3.037, CEP 37200-000 Lavras, MG. \\ E-mail: vanessaforesti@yahoo.com.br, mlucio@ufla.br, ribeirojuniorpm@yahoo.com.br, rodolphovitorino@hotmail.com (2)Empresa \\ de Pesquisa Agropecuária de Minas Gerais, Núcleo Tecnológico Epamig Uva e Vinho, Avenida Santa Cruz, no-500, Caixa Postal 33, \\ CEP 37780-000 Caldas, MG. E-mail: murillo@epamigcaldas.gov.br, renata@epamigcaldas.gov.br
}

Resumo - O objetivo deste trabalho foi avaliar o efeito de doses crescentes de duas formulações de fosfito de potássio, sobre a severidade do míldio da videira, produtividade e características físico-químicas de uvas Merlot. Foram avaliadas duas formulações de fosfito de potássio (Phi A e B), nas doses 0, 1,2, 2,5, 3,7 e 5,0 $\mathrm{g} \mathrm{L}^{-1}$ de $\mathrm{P}_{2} \mathrm{O}_{5}$, comparadas com um fertilizante foliar e fungicidas, nas safras de 2009/2010 e 2010/2011. Observou-se efeito linear das doses de fosfito sobre a redução da severidade do míldio, nas duas safras. A maior dose de Phi A proporcionou controle médio de $60,5 \%$ e a de Phi B de 57,7\%, semelhante ao controle pelos fungicidas $(64,3 \%)$ e pelo fertilizante foliar $(53,3 \%)$. Na primeira safra, Phi B proporcionou maior produtividade e, na segunda, não diferiu do tratamento com o fungicida. Os tratamentos somente produziram efeito sobre teor de sólidos solúveis, $\mathrm{pH}$ e acidez total titulável do mosto na segunda safra. Phi B e fungicidas proporcionaram os maiores teores de compostos fenólicos solúveis totais das cascas, porém, não produziram efeito sobre os teores de antocianinas. A aplicação de fosfito de potássio aumenta o teor de sólidos solúveis totais e o $\mathrm{pH}$, reduz a acidez total titulável das bagas, não afeta o teor de antocianinas e é alternativa eficaz para o controle do míldio da videira, com resposta similar à proporcionada por fungicidas.

Termos para indexação: Plasmopara viticola, Vitis vinifera, compostos fenólicos, controle alternativo.

\section{Potassium phosphite on the control of downy mildew of grapevine and physicochemical characteristics of Merlot grapes}

\begin{abstract}
The objective of this work was to evaluate the effect of increasing doses of two formulations of potassium phosphite, on the severity of downy mildew of grapevine, yield and on physicochemical characteristics of Merlot grapes. Two formulations of potassium phosphite (Phi A and B) were evaluated at the doses $0,1.2,2.5,3.7$ and $5.0 \mathrm{~g} \mathrm{~L}^{-1}$ of $\mathrm{P}_{2} \mathrm{O}_{5}$, in comparison with a foliar fertilizer and fungicides, during the 2009/2010 and 2010/2011 crop seasons. There was a linear effect of phosphite doses on the reduction of the downy mildew severity, in the two crop seasons. The Phi A highest dose provided an average control of $60.5 \%$, and Phi B, 57.7\%, similar to the control by the fungicides (64.3\%) and fertilizer (53.3\%). In the first harvest, Phi B provided a greater productivity, while, in the second, it did not differ from the treatment with fungicides. Treatments only affected soluble solids, $\mathrm{pH}$ and total acidity of the grape must in the second season. Phi B and fungicides provided the highest levels of total soluble phenolic compounds at the grape peel, however, had no effect on the levels of anthocyanins. Potassium phosphite application increases total soluble solids and $\mathrm{pH}$, reduces total titratable acidity of the berries, does not affect anthocyanin contents, and it is an effective alternative for the control of downy mildew, with response similar to that of fungicides.
\end{abstract}

Index terms: Plasmopara viticola, Vitis vinifera, phenolic compounds, alternative control.

\section{Introdução}

O míldio, causado pelo oomiceto Plasmopara viticola (Berk.\& Curt) Berl. \& de Toni, é uma das doenças mais prejudiciais aos vinhedos. Esse patógeno pode infectar todos os órgãos vegetativos da videira, e torna-se crítico quando a infecção ocorre no início do florescimento e na frutificação, podendo causar severa redução da produtividade (Sônego \& Garrido, 2005; Hofmann, 2008).

O método mais utilizado pelos viticultores, para o controle do míldio, consiste na aplicação de fungicidas específicos, de contato e sistêmicos (Sônego \& Garrido, 2005). Entretanto, estes produtos podem levar à seleção de populações resistentes aos princípios 
ativos utilizados (Madden et al., 2000). Além disso, sua intensa aplicação pode acarretar contaminação ambiental e das uvas, intoxicação dos agricultores, prejuízo à fermentação alcoólica (por afetar a população das leveduras), além de aumentar o custo de produção (Sônego \& Garrido, 2005; González Álvarez et al., 2012).

Dessa forma, alternativas capazes de auxiliar no controle de doenças, mas que não representem risco ao homem e ao meio ambiente (Stadinik \& Talamini, 2004), têm grande relevância. Os fosfitos têm sido utilizados para o controle de doenças de diversas culturas, tais como ferrugem da videira, ferrugem e oídio do trigo, míldio da soja, podridão em maçãs, e apresentado resultados promissores no controle também de outros patógenos, inclusive o míldio da videira (Sônego \& Garrido, 2005; Sautter et al., 2008; Pereira et al., 2010; Gomes et al., 2011; Santos et al., 2011; Silva et al., 2011).

Gomes et al. (2011) observaram que plantas da cultivar de videira Isabel, tratadas com fosfito de potássio (130 g $\left.100 \mathrm{~L}^{-1}\right)$, apresentaram $11,47 \%$ de redução da incidência do míldio e produtividade média de 3,83 $\mathrm{Mg} \mathrm{ha}^{-1}$ de uvas, semelhantemente ao tratamento com fungicidas. Peruch \& Bruna (2008) observaram redução de $76 \%$ da incidência de míldio em cachos da cultivar Goethe. Na maioria dos estudos, o íon fosfito parece ter ação direta sobre o patógeno; contudo, alguns autores relatam que ele também apresenta ação indutora de resistência nas plantas. A indução de resistência pode ocorrer pela produção de fitoalexinas, acúmulo de lignina, fenóis solúveis e ativação de outros compostos de defesa das plantas (Jackson et al., 2000; Daniel \& Guest, 2005; Nojosa et al., 2005; Sautter et al., 2008).

Os compostos fenólicos são componentes importantes nos derivados da uva, como sucos e vinhos, e contribuem para a expressão de características sensoriais como cor, sabor, adstringência e estrutura do vinho, por ação direta ou por interação com proteínas, polissacarídeos ou outros compostos fenólicos (Abe et al., 2007). Em uvas tintas, as antocianinas constituem a maior percentagem de compostos fenólicos e contribuem para os atributos sensoriais e, principalmente, para a coloração do vinho e do mosto de uvas (Muñoz-Espada et al., 2004; Abe et al., 2007; Brunetto et al., 2007). Além de essenciais para a pigmentação dos vegetais, os compostos fenólicos também apresentam ação contra fitopatógenos (Iriti et al., 2011).

Algumas doenças da videira, como o míldio, podem influenciar a qualidade físico-química das bagas, ao interferir na síntese de compostos fenólicos (Sônego et al., 2005; Gil \& Pszczolkocwski, 2007). Dessa forma, produtos utilizados no seu controle também podem influenciar a qualidade final das uvas e de seus derivados (Dugo et al., 2004; Cuš \& Raspor, 2008; González Álvarez et al., 2012).

O objetivo deste trabalho foi avaliar o efeito de doses crescentes de duas formulações de fosfitos de potássio sobre a severidade do míldio da videira, produtividade e características físico-químicas de uvas Merlot.

\section{Material e Métodos}

O experimento foi realizado em duas safras, 2009/2010 e 2010/2011, em vinhedo localizado no Núcleo Tecnológico da Epamig Uva e Vinho, em Caldas, MG, a $21^{\circ} 55^{\prime} 25^{\prime \prime} \mathrm{S}, 46^{\circ} 23^{\prime} 10^{\prime \prime} \mathrm{W}$ e a $1.150 \mathrm{~m}$ de altitude. Foram utilizadas plantas da cultivar Merlot (Clone 343) de Vitis vinifera, com seis anos de idade, enxertadas sobre o porta-enxerto 1103 Paulsen, em espaçamento de $2,5 \times 1,5 \mathrm{~m}$ e condução em espaldeira com três fios de arame. As plantas foram conduzidas em duplo cordão esporonado (sistema Royat), podadas com poda curta (duas gemas), tendo permanecido com 20 a 22 gemas por planta, o que resultou em um total de 50 a 55 mil gemas por hectare.

Os tratamentos consistiram da aplicação do fertilizante foliar Fulland (Sudoeste Fertilizantes Ltda., Araxá, MG, Brasil), dos fosfitos de potássio Phi A, Pepfós, (Pepita Fertilizantes Ltda., Poços de Caldas, MG) e Phi B, Reforce, (Agrichem do Brasil Ltda., Ribeirão Preto, SP), do tratamento químico com fungicidas, e da testemunha, sem nenhum tipo de pulverização. $\mathrm{O}$ fertilizante foliar tinha a seguinte formulação: $46,55 \mathrm{~g} \mathrm{~L}^{-1}$ de $\mathrm{Cu}, 266 \mathrm{~g} \mathrm{~L}^{-1}$ de $\mathrm{P}_{2} \mathrm{O}_{5} \mathrm{e}$ $23,27 \mathrm{~g} \mathrm{~L}^{-1}$ de S. Os fosfitos apresentavam $280 \mathrm{~g} \mathrm{~L}^{-1}$ de $\mathrm{K}_{2} \mathrm{O}$ e $420 \mathrm{~g} \mathrm{~L}^{-1}$ de $\mathrm{P}_{2} \mathrm{O}_{5}$ (Phi A) ou $240 \mathrm{~g} \mathrm{~L}^{-1}$ de $\mathrm{K}_{2} \mathrm{O}$ e $340 \mathrm{~g} \mathrm{~L}^{-1}$ de $\mathrm{P}_{2} \mathrm{O}_{5}$ (Phi B). O controle químico foi feito com emprego alternado dos fungicidas Cabrio Top - $550 \mathrm{~g} \mathrm{~kg}^{-1}$ metiram e $50 \mathrm{~g} \mathrm{~kg}^{-1}$ piraclostrobina-, (Basf S.A., São Paulo, SP), Daconil - $500 \mathrm{~g} \mathrm{~L}^{-1}$ clorotalonil -, (Syngenta Proteção de Cultivos Ltda., Paulínia, SP) e Ridomil - $40 \mathrm{~g} \mathrm{~kg}^{-1}$ metalaxil-M e 
$640 \mathrm{~g} \mathrm{~kg}^{-1}$ mancozeb -, (Syngenta Proteção de Cultivos Ltda., Paulínia, SP).

Foram utilizadas cinco doses de cada fosfito: 0 , $0,525,1,05,1,58$ e $2,1 \mathrm{~g} \mathrm{~L}^{-1}$ de $\mathrm{P}_{2} \mathrm{O}_{5}$. De acordo com a dose de $\mathrm{P}_{2} \mathrm{O}_{5}$, utilizou-se para $\mathrm{Phi} A$ as doses $0,1,25$, 2,5, 3,76 e 5,0 $\mathrm{mL} \mathrm{L}^{-1}$ e, para Phi $\mathrm{B}$, as doses $0,1,5$, 3,0, 4,5 e 6,0 $\mathrm{mL} \mathrm{L}^{-1}$. O fertilizante foliar Fulland foi aplicado à dose de $5,9 \mathrm{~mL} \mathrm{~L}^{-1}$, e o tratamento químico consistiu da pulverização, de forma alternada, dos fungicidas Cabrio Top $\left(2 \mathrm{~g} \mathrm{~L}^{-1}\right)$, Daconil $\left(2 \mathrm{~g} \mathrm{~L}^{-1}\right) \mathrm{e}$ Ridomil $\left(2,5 \mathrm{~g} \mathrm{~L}^{-1}\right)$. As pulverizações foram realizadas a partir da brotação das videiras, em intervalos semanais, no total de 12 aplicações, realizadas com pulverizador costal manual até o ponto de escorrimento. Foi adicionado $0,05 \%$ de óleo mineral à calda de pulverização, e o volume aplicado variou de 700 a $1.000 \mathrm{~L} \mathrm{ha}^{-1}$, de acordo com o estágio de desenvolvimento da planta.

A avaliação da severidade do míldio foi feita nas folhas, quinzenalmente, desde o surgimento dos primeiros sintomas. Nas duas safras, foram realizadas cinco avaliações até o momento da colheita, com início em 5/11/2009, na safra 2009/2010, e em 18/11/2010 na safra 2010/2011. Foi utilizada a escala de notas proposta por Azevedo (1997), com algumas modificações. A severidade foi avaliada em toda a planta, dentro da parcela útil, com notas que variaram de 0 a 5 , das quais a nota 0 foi atribuída a plantas com ausência de sintomas, e as notas de 1 a 5 conforme a área foliar lesionada: 1 , de 0,1 a $5 \%$; 2 , de 5,1 a $25 \%$; 3 , de 25,1 a $35 \%$; 4, de 35,1 a $50 \%$; e 5 , mais de $50 \%$. A partir dos resultados das avaliações, foram calculados os níveis de severidade e a área abaixo da curva de progresso da severidade do míldio da videira (AACPSD), para cada tratamento. Os índices de controle do míldio foram obtidos pela relação 1 - (x/y), em que x representa o índice da doença das plantas tratadas, e y representa o índice de doença da testemunha sem pulverização ( $\mathrm{Li}$ et al., 1996).

Nas duas safras, além da quantificação da severidade do míldio, foram avaliadas a produtividade e a qualidade da baga, em relação às doses dos fosfitos que apresentaram maior controle do míldio, e em relação aos tratamentos com fertilizante foliar e fungicidas. A produtividade e a estimativa de produção foram determinadas quanto a: massa de cachos $(\mathrm{kg})$ por parcela; número total de cachos por parcela; número total de cachos no estádio fenológico pintor; e massa média de cachos e bagas, expressos em gramas. $\mathrm{O}$ teor de sólidos solúveis totais ( ${ }^{\circ} \mathrm{Brix}$ ) foi determinado em refratômetro digital portátil Pal 1 Atago (Atago Co., Ltd., Saitama, Japão); a acidez total titulável, por meio de titulação com $\mathrm{NaOH} 0,1 \mathrm{~N}$, com fenolftaleína como indicador, conforme técnica descrita em Elrich (1990); e a avaliação do $\mathrm{pH}$ foi feita por meio de medidor de pH modelo B-474 (Micronal, São Paulo, SP, Brasil), calibrado com padrões 4,0 e 7,0.

Na segunda safra, de 2010/2011, além das avaliações mencionadas, as cascas de 150 bagas por tratamento, por parcela, foram separadas, pesadas, congeladas em nitrogênio líquido e armazenadas a $-80^{\circ} \mathrm{C}$, para a determinação do teor de antocianinas e fenólicos totais. Para a determinação dos compostos fenólicos, $150 \mathrm{mg}$ de casca triturada em nitrogênio líquido foram homogeneizados em IKA T-18 basic Ultra Turrax (IKA Works, Inc., Artur Nogueira, SP, Brasil), em solução extratora constituída de metanol acidificado ( $\mathrm{HCl} 1 \%)$. As antocianinas foram determinadas pelo método do pH diferencial (Giusti \& Wrolstad, 2000). Os fenólicos totais foram analisados pelo método de Folin-Ciocalteau, com base em uma curva-padrão de ácido gálico (Amerine \& Ough, 1980).

O delineamento experimental, utilizado na avaliação da produtividade e qualidade, foi o de blocos ao acaso, em arranjo fatorial $2 \times 5+2$ (dois produtos, cinco doses e dois tratamentos adicionais com aplicação de fungicidas ou do fertilizante foliar), com quatro repetições de seis plantas em linha por parcela, e parcela útil formada pelas quatro plantas centrais da linha de plantio. Utilizou-se bordadura de uma linha, entre as linhas que receberam pulverização com os tratamentos. Análises de regressão foram utilizadas entre as doses dos fosfitos de potássio e os dados de AACPSD. Os tratamentos adicionais foram comparados às doses dos fosfitos por meio do teste de Dunnett, a 5\% de probabilidade, por meio do programa SAS v. 9.0. Os dados de produtividade e de características físico-químicas dos frutos foram submetidos à análise de variância pelo programa Sisvar. As médias, quando significativas pelo teste F, foram comparadas pelo teste de Scott-Knott, a 5\% de probabilidade.

\section{Resultados e Discussão}

Observou-se efeito das doses dos fosfitos de potássio avaliados na área abaixo da curva do progresso da 
severidade do míldio (AACPSD) (Figura 1). As duas formulações de fosfito utilizadas diminuíram a AACPSD de forma linear, com o aumento das doses. As doses com maior concentração de $\mathrm{P}_{2} \mathrm{O}_{5}\left(2,1 \mathrm{~g} \mathrm{~L}^{-1}\right.$ de $\mathrm{P}_{2} \mathrm{O}_{5}$ ) proporcionaram maior redução da severidade do míldio da videira, nas duas safras avaliadas, com controle médio de $60,5 \%$ para fosfito Phi A, e $57,7 \%$ para $\mathrm{Phi} \mathrm{B}$, em comparação à testemunha sem pulverização.

Na primeira safra, de 2009/2010, os tratamentos com menor dose de Phi A $\left(0,525 \mathrm{~g} \mathrm{de}_{2} \mathrm{O}_{5} \mathrm{~L}^{-1}\right)$ e a testemunha (dose $0 \mathrm{~g}$ ) apresentaram maior severidade do míldio da videira que o tratamento controle com fungicidas e o com fertilizante foliar Fulland (Tabela 1). Entretanto, as doses maiores de Phi A proporcionaram controle entre 42,4 e $61,8 \%$, estatisticamente semelhante ao obtido com o tratamento padrão com fungicidas $(63,5 \%)$ e com fertilizante foliar Fulland $(58,4 \%)$. Para o tratamento com Phi B, apenas a dose com maior teor de $\mathrm{P}_{2} \mathrm{O}_{5}\left(2,1 \mathrm{~g} \mathrm{~L}^{-1}\right)$ reduziu significativamente a severidade do míldio, sem diferença para o tratamento com fungicidas. Quando os tratamentos com Phi B foram comparados ao do fertilizante foliar, apenas a menor dose causou maior severidade da doença.
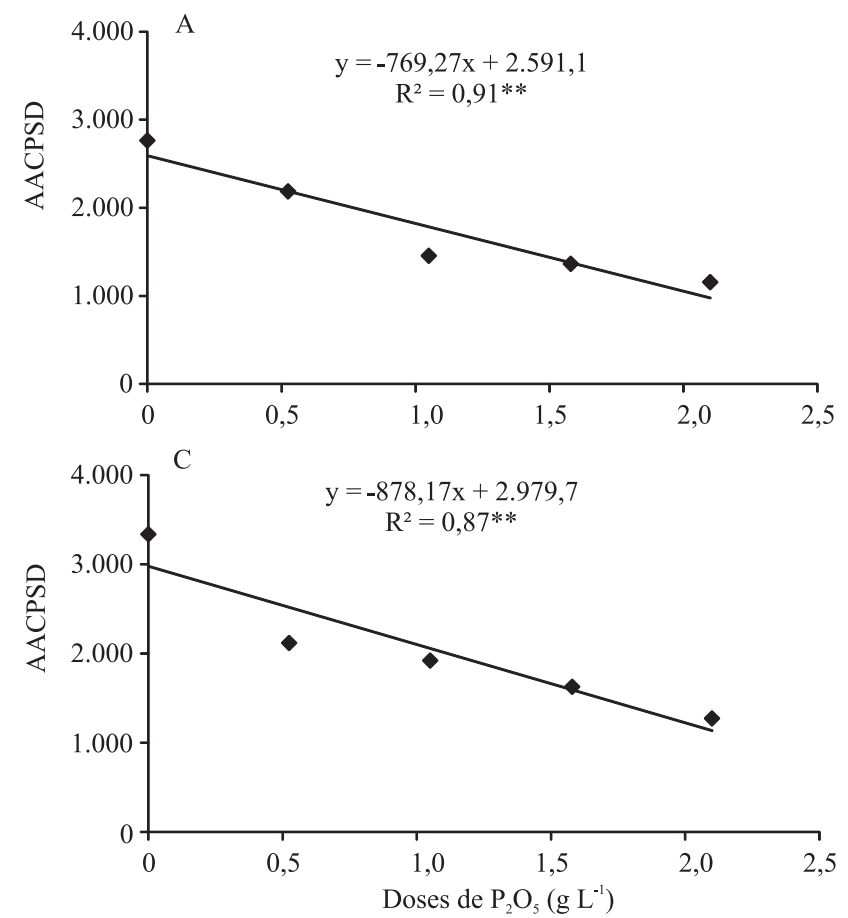

Nas demais doses, esse fosfito combateu a severidade do míldio de modo semelhante ao do tratamento com o fertilizante, com controle da doença de 47,4 a 55,0\%.

Na segunda safra (2010/2011), os fosfitos Phi A e Phi B proporcionaram controle da doença semelhante ao obtido com o controle padrão, de aplicação alternada de diferentes fungicidas, quando foram utilizadas doses de $\mathrm{P}_{2} \mathrm{O}_{5}$ de $1,58 \mathrm{~L}^{-1}$ para Phi A e 2,1g L ${ }^{-1}$ para Phi B. Vários trabalhos relatam a eficiência de fosfitos de potássio no controle de doenças de plantas, além de seu duplo modo de ação - tóxica na aplicação direta sobre os patógenos e em atuação indireta, pela ativação de mecanismos de defesa nas plantas (Jackson et al., 2000; Nojosa et al., 2005; Sônego \& Garrido, 2005; Pereira et al., 2010; Santos et al., 2011).

Silva et al. (2011), ao avaliar doses de fosfitos de potássio no controle do míldio da soja, observaram que o aumento de teores de fosfito $(750$ e $1500 \mathrm{~g}$ de $\left.\mathrm{P}_{2} \mathrm{O}_{5}-30 \% \mathrm{P}_{2} \mathrm{O}_{5}+20 \% \mathrm{~K}_{2} 0\right)$ resultou em controle superior a $50 \%$ da doença, além de maior rendimento de grãos e menor incidência de patógenos nas sementes. Pereira et al. (2010) observaram que diferentes fontes de fosfito de potássio, a $6,0 \mathrm{~mL} \mathrm{~L}^{-1}$, proporcionaram controle entre 56 e $80 \%$ do míldio em folhas da cultivar
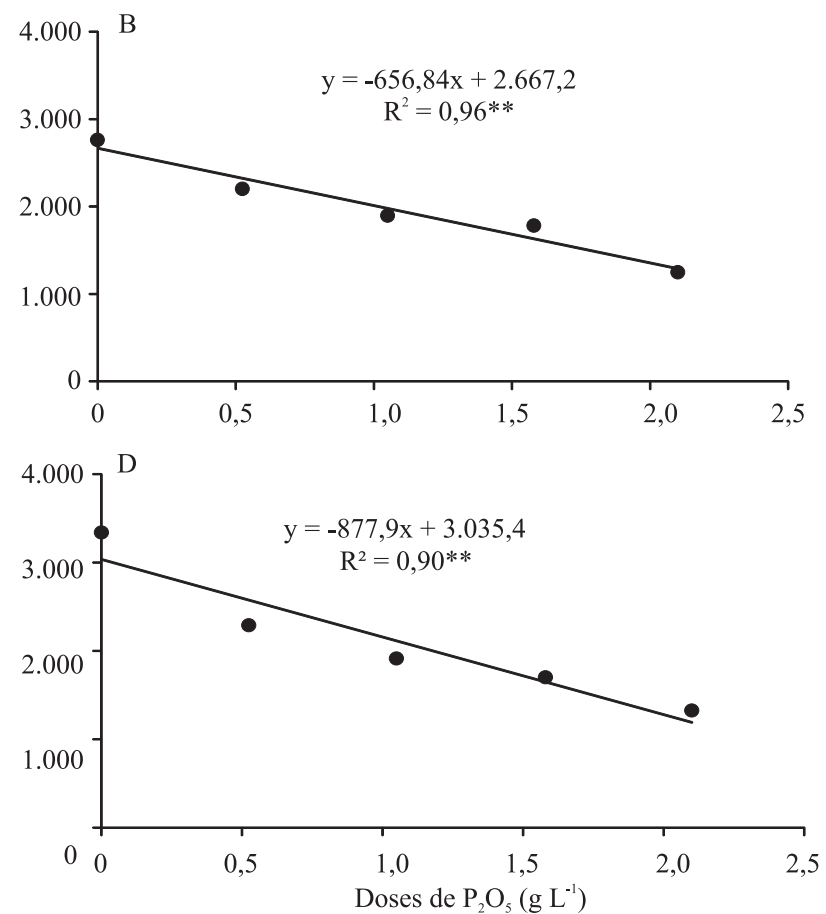

Figura 1. Efeito das doses avaliadas dos fosfitos de potássio Phi A ( A e C) e Phi B (B e D) - com base nos teores de $\mathrm{P}_{2} \mathrm{O}_{5}$ de cada produto - na área abaixo da curva de severidade do míldio (AACPSD), em folhas de videira 'Merlot', durante as safras 2009/2010 (A e B) e 2010/2011 (C e D). **Significativo, pelo teste F, a 5\% de probabilidade. 
de videira Merlot, em comparação à testemunha sem pulverização.

Nas avaliações de produtividade e análises físico-químicas das bagas, na primeira safra, o Phi B destacou-se por ter proporcionado maior produtividade e massa média de cachos e bagas (Tabela 2). Na segunda safra, o tratamento Phi B não diferiu estatisticamente do tratamento com fungicidas e proporcionou maior produtividade, e maior massa de cachos e bagas que a testemunha, o Phi A e o fertilizante foliar. No entanto, para o número de cachos colhidos, todos os tratamentos foram estatisticamente semelhantes ao fungicida e superiores à testemunha.

$\mathrm{Na}$ safra 2009/2010, a produtividade da videira foi baixa, o que pode estar relacionado a condições climáticas desfavoráveis verificadas, principalmente, no período de inflorescência, quando foram registrados $127,9 \mathrm{~mm}$ de chuva, concentrados em apenas três dias, com alta umidade relativa média (82\%). Na fase final de maturação, no entanto, ocorreu alto índice pluvial (183 mm), alta umidade relativa do ar (84\%) e poucas horas de insolação (46 horas), o que elevou as perdas na colheita. Em 2010/2011, as condições climáticas foram favoráveis à maturação das uvas (temperaturas médias máximas em torno de $28,1^{\circ} \mathrm{C}$, pluviosidade média de $97,8 \mathrm{~mm}$, umidade relativa do ar em torno de $80 \%$ e período de insolação de 105 horas), o que permitiu maturação fenólica próxima das condições ideais para colheita, além de maior produtividade.

Peruch \& Bruna (2008) não observaram aumento na produtividade da cultivar de videira Goethe, com o aumento de doses de fosfitos de potássio, apesar de terem obtido redução de $94 \%$ da severidade do míldio nas folhas e de $76 \%$ da incidência de doença nos cachos. Silva et al. (2011) verificaram rendimento da soja tratada com fosfito de potássio semelhante

Tabela 1. Efeito das doses avaliadas dos fosfitos de potássio Phi A e Phi B - com base nos teores de $\mathrm{P}_{2} \mathrm{O}_{5}$ de cada produto -, na área abaixo da curva de severidade do míldio (AACPSD), em folhas de videira 'Merlot', durante as safras 2009/2010 e 2010/2011, em comparação aos tratamentos controle com fungicidas e fertilizante foliar.

\begin{tabular}{|c|c|c|c|c|c|c|}
\hline \multirow[t]{2}{*}{ Produtos $^{(1)}$} & \multirow{2}{*}{$\begin{array}{c}\mathrm{P}_{2} 0_{5} \\
\left(\mathrm{~g} \mathrm{~L}^{-1}\right)\end{array}$} & \multirow{2}{*}{$\begin{array}{c}\text { Doses } \\
\left(\mathrm{mL} \mathrm{L}^{-1}\right)\end{array}$} & \multirow[t]{2}{*}{ AACPSD } & \multicolumn{2}{|c|}{ Significância $^{(2)}$} & \multirow{2}{*}{$\begin{array}{c}\text { Controle } \\
(\%)\end{array}$} \\
\hline & & & & Fungicida & Fertilizante & \\
\hline & \multicolumn{6}{|c|}{ Safra 2009/2010 } \\
\hline Testemunha & 0,0 & - & $2.762,5$ & $*$ & $*$ & - \\
\hline Phi A & 0,525 & 1,25 & $2.183,6$ & $*$ & * & 21,0 \\
\hline Phi A & 1,05 & 2,5 & $1.452,9$ & & & 47,4 \\
\hline Phi A & 1,58 & 3,76 & $1.361,3$ & & & 50,7 \\
\hline Phi A & 2,1 & 5,0 & $1.152,5$ & & & 58,3 \\
\hline Phi B & 0,525 & 1,5 & $2.200,0$ & $*$ & $*$ & 20,4 \\
\hline Phi B & 1,05 & 3,0 & $1.895,0$ & $*$ & & 31,4 \\
\hline Phi B & 1,58 & 4,5 & $1.781,9$ & $*$ & & 35,5 \\
\hline Phi B & 2,1 & 6,0 & $1.245,0$ & & & 55,0 \\
\hline Fungicida & & & 965,0 & - & & 65,1 \\
\hline \multirow[t]{2}{*}{ Fertilizante } & & & $1.431,6$ & & - & 48,2 \\
\hline & \multicolumn{6}{|c|}{ Safra $2010 / 2011$} \\
\hline Testemunha & 0,0 & - & $3.337,3$ & $*$ & $*$ & - \\
\hline Phi A & 0,525 & 1,25 & $2.119,8$ & $*$ & $*$ & 36,5 \\
\hline Phi A & 1,05 & 2,5 & $1.922,4$ & $*$ & & 42,4 \\
\hline Phi A & 1,58 & 3,76 & $1.629,1$ & & & 51,2 \\
\hline Phi A & 2,1 & 5,0 & $1.218,5$ & & & 61,8 \\
\hline Phi B & 0,525 & 1,5 & $2.288,3$ & $*$ & $*$ & 31,4 \\
\hline Phi B & 1,05 & 3,0 & $1.913,4$ & $*$ & & 42,7 \\
\hline Phi B & 1,58 & 4,5 & $1.700,0$ & & & 49,1 \\
\hline Phi B & 2,1 & 6,0 & $1.324,5$ & & & 60,3 \\
\hline Fungicida & & & $1.218,5$ & - & & 63,5 \\
\hline$\underline{\text { Fertilizante }}$ & & & $1.388,3$ & & - & 58,4 \\
\hline
\end{tabular}

${ }^{(1)} \mathrm{Phi}$ A, fosfito de potássio com formulação de $420 \mathrm{~g} \mathrm{~L}^{-1}$ de $\mathrm{P}_{2} \mathrm{O}_{5}$ e $280 \mathrm{~g} \mathrm{~L}^{-1}$ de $\mathrm{K}_{2} \mathrm{O}$; Phi $\mathrm{B}$, fosfito de potássio com formulação de $340 \mathrm{~g} \mathrm{~L}{ }^{-1}$ de $\mathrm{P}_{2} \mathrm{O}_{5}$ e $240 \mathrm{~g} \mathrm{~L}^{-1}$ de $\mathrm{K}_{2} \mathrm{O}$; Fungicida, aplicação alternada de Cabrio Top, Daconil e Ridomil; Fertilizante, aplicação do fertilizante foliar Fulland; Testemunha, plantas sem pulverização. ${ }^{(2)}$ Significativo, pelo teste de Dunnett, a 5\% de probabilidade, na comparação com o tratamento com fungicidas, na primeira coluna, e com fertilizante foliar Fulland, na segunda coluna. 
ao de plantas tratadas com fungicida. Gomes et al. (2011) verificaram que fosfitos de potássio (130 g por $100 \mathrm{~L}$ ) proporcionaram produtividade semelhante $\mathrm{e}$ até melhor do que a de plantas tratadas com fungicidas, em videiras 'Isabel'; porém, os autores relataram aumento da acidez titulável e redução do $\mathrm{pH}$ do mosto.

Quanto às características analíticas das bagas, na primeira safra não se observou efeito significativo dos tratamentos sobre o teor de sólidos solúveis, $\mathrm{pH}$ e acidez total titulável do mosto (Tabela 3). Entretanto, na segunda safra, todos os tratamentos avaliados proporcionaram mudanças nesses parâmetros, sem diferirem entre si, mas superiores à testemunha. Pereira et al. (2010) também não constataram diferenças na qualidade analítica de bagas tratadas com fosfitos. No entanto, Gomes et al. (2011) observaram que videiras 'Isabel' tratadas com fosfito de potássio (130 g $\left.100 \mathrm{~L}^{-1}\right)$ e bioflavonoides cítricos apresentaram bagas com maior teor de sólidos solúveis e menor $\mathrm{pH}$ que os observados no tratamento sem pulverização.

A severidade do míldio pode alterar o metabolismo da videira e ocasionar menor acúmulo de metabólitos nos frutos, o que resultaria em menor teor de sólidos solúveis totais (Mullins et al., 1992). Além disso, a alta incidência da doença e o excesso de chuva, no período de maturação das uvas, pode levar à podridão das bagas, o que acarreta maior ocorrência de insetos e micro-organismos, como bactérias, além de favorecer o aumento da acidez do mosto (Conde et al., 2007).

Tabela 2. Efeito dos tratamentos sobre a produtividade, número de cachos colhidos e massa média de cachos e de bagas da videira 'Merlot'(1).

\begin{tabular}{|c|c|c|c|c|}
\hline Tratamento $^{(2)}$ & $\begin{array}{l}\text { Produtividade } \\
\text { (kg por parcela) }\end{array}$ & $\begin{array}{l}\text { Cachos } \\
\text { colhidos }\end{array}$ & $\begin{array}{l}\text { Massa média } \\
\text { de cachos (g) }\end{array}$ & $\begin{array}{c}\text { Massa } \\
\text { de bagas }(\mathrm{g})\end{array}$ \\
\hline & \multicolumn{4}{|c|}{ Safra 2009/2010 } \\
\hline Testemunha & $0,10 \mathrm{e}$ & $11,5 \mathrm{a}$ & $8,9 \mathrm{c}$ & $1,4 \mathrm{~b}$ \\
\hline Fungicidas & $0,81 b$ & $16,3 \mathrm{a}$ & $53,5 \mathrm{a}$ & $1,5 \mathrm{~b}$ \\
\hline Fertilizante & $0,38 \mathrm{~d}$ & $15,0 \mathrm{a}$ & $25,3 b$ & $1,6 b$ \\
\hline Phi A $\left(2,1 \mathrm{~g} \mathrm{~L}^{-1}\right.$ de $\left.\mathrm{P}_{2} \mathrm{O}_{5}\right)$ & $0,61 \mathrm{c}$ & $16,3 \mathrm{a}$ & $37,6 b$ & $1,6 b$ \\
\hline \multirow[t]{2}{*}{ Phi B $\left(2,1 \mathrm{~g} \mathrm{~L}^{-1}\right.$ de $\left.\mathrm{P}_{2} \mathrm{O}_{5}\right)$} & $0,93 \mathrm{a}$ & $14,3 \mathrm{a}$ & $67,5 \mathrm{a}$ & $2,1 \mathrm{a}$ \\
\hline & \multicolumn{4}{|c|}{ Safra 2010/2011 } \\
\hline Testemunha & $0,16 \mathrm{c}$ & $20,0 b$ & $7,9 \mathrm{c}$ & $0,7 \mathrm{c}$ \\
\hline Fungicidas & $2,67 \mathrm{a}$ & $41,5 \mathrm{a}$ & $65,3 \mathrm{a}$ & $1,5 \mathrm{a}$ \\
\hline Fertilizante & $1,29 b$ & $42,0 \mathrm{a}$ & $33,7 b$ & $1,2 \mathrm{~b}$ \\
\hline Phi A $\left(2,1 \mathrm{~g} \mathrm{~L}^{-1}\right.$ de $\left.\mathrm{P}_{2} \mathrm{O}_{5}\right)$ & $1,52 b$ & $44,5 \mathrm{a}$ & $28,9 \mathrm{~b}$ & $1,3 \mathrm{~b}$ \\
\hline Phi B $\left(2,1 \mathrm{~g} \mathrm{~L}^{-1}\right.$ de $\left.\mathrm{P}_{2} \mathrm{O}_{5}\right)$ & $1,93 \mathrm{a}$ & $47,5 \mathrm{a}$ & $47,4 \mathrm{a}$ & $1,4 \mathrm{a}$ \\
\hline
\end{tabular}

(1)Médias seguidas de letras iguais, nas colunas, não diferem pelo teste Scott-Knott, a $5 \%$ de probabilidade. ${ }^{(2)} \mathrm{Phi} \mathrm{A}$, fosfito de potássio com formulação de $420 \mathrm{~g} \mathrm{~L}^{-1}$ de $\mathrm{P}_{2} \mathrm{O}_{5}$ e $280 \mathrm{~g} \mathrm{~L}^{-1}$ de $\mathrm{K}_{2} \mathrm{O}$; Phi B, fosfito de potássio com formulação de $340 \mathrm{~g} \mathrm{~L}^{-1}$ de $_{2} \mathrm{O}_{5}$ e $240 \mathrm{~g} \mathrm{~L}^{-1}$ de $\mathrm{K}_{2} \mathrm{O}$; Fungicida, aplicação alternada de Cabrio Top, Daconil e Ridomil; Fertilizante, aplicação do fertilizante foliar Fulland; Testemunha, plantas sem pulverização.
Não foram observadas diferenças significativas entre os tratamentos, quanto ao teor de antocianinas totais; porém, nos tratamentos com fungicida e Phi B, o teor de fenóis solúveis totais nas cascas foi maior que o dos tratamentos com fertilizante foliar e Phi A (Tabela 4).

Os metabólitos secundários, tais como compostos fenólicos e fitoalexinas, são acumulados nos tecidos vegetais em resposta à infecção por patógenos, estresses abióticos e, também, pela aplicação de produtos capazes de induzir resistência sistêmica nas plantas, o que acarretaria maior acúmulo de metabólitos de defesa (Hukkanem et al., 2007; Iriti et al., 2011).

O modo de ação do ânion fosfito nas plantas tem sido relacionado ao aumento da resposta de

Tabela 3. Efeito dos tratamentos sobre características analíticas de bagas de uva 'Merlot'(1).

\begin{tabular}{lccc}
\hline Tratamento $^{(2)}$ & $\begin{array}{c}\text { Sólidos solúveis } \\
\left.\text { totais ( }{ }^{\circ} \mathrm{Brix}\right)\end{array}$ & $\mathrm{pH}$ & $\begin{array}{c}\text { Acidez titulável } \\
\left(\mathrm{g} \mathrm{L}^{-1}\right)\end{array}$ \\
\hline & \multicolumn{3}{c}{ Safra 2009/2010 } \\
Testemunha & 15,9 & 3,3 & 9,1 \\
Fungicidas & 16,4 & 3,2 & 9,1 \\
Fertilizante & 16,4 & 3,2 & 9,6 \\
Phi A $\left(2,1 \mathrm{~g} \mathrm{~L}^{-1} \mathrm{P}_{2} \mathrm{O}_{5}\right)$ & 16,1 & 3,2 & 9,0 \\
Phi B $\left(2,1 \mathrm{~g} \mathrm{~L}^{-1} \mathrm{P}_{2} \mathrm{O}_{5}\right)$ & 15,8 & 3,2 & 8,9 \\
\hline & \multicolumn{3}{c}{ Safra 2010/2011 } \\
Testemunha & $18,0 \mathrm{~b}$ & $2,8 \mathrm{~b}$ & $9,8 \mathrm{~b}$ \\
Fungicidas & $19,2 \mathrm{a}$ & $3,3 \mathrm{a}$ & $8,2 \mathrm{a}$ \\
Fertilizante & $19,5 \mathrm{a}$ & $3,4 \mathrm{a}$ & $8,6 \mathrm{a}$ \\
Phi A $\left(2,1 \mathrm{~g} \mathrm{~L}^{-1} \mathrm{P}_{2} \mathrm{O}_{5}\right)$ & $18,8 \mathrm{a}$ & $3,5 \mathrm{a}$ & $8,4 \mathrm{a}$ \\
Phi B $\left(2,1 \mathrm{~g} \mathrm{~L}^{-1} \mathrm{P}_{2} \mathrm{O}_{5}\right)$ & $19,1 \mathrm{a}$ & $3,5 \mathrm{a}$ & $8,6 \mathrm{a}$ \\
\hline
\end{tabular}

(1)Médias seguidas de letras iguais, nas colunas, não diferem pelo teste ScottKnott, a $5 \%$ de probailidade. ${ }^{(2)} \mathrm{Phi} \mathrm{A}$, fosfito de potássio com formulação de $420 \mathrm{~g} \mathrm{~L}^{-1}$ de $\mathrm{P}_{2} \mathrm{O}_{5}$ e $280 \mathrm{~g} \mathrm{~L}^{-1}$ de $\mathrm{K}_{2} \mathrm{O}$; Phi B, fosfito de potássio com formulação de $340 \mathrm{~g} \mathrm{~L}^{-1}$ de $\mathrm{P}_{2} \mathrm{O}_{5}$ e $240 \mathrm{~g} \mathrm{~L}^{-1}$ de $\mathrm{K}_{2} \mathrm{O}$; Fungicida, aplicação alternada de Cabrio Top, Daconil e Ridomil; Fertilizante, aplicação do fertilizante foliar Fulland; Testemunha, plantas sem pulverização.

Tabela 4. Efeito dos tratamentos sobre os teores de antocianinas e de compostos fenólicos solúveis totais, em cascas de uva 'Merlot', na safra 2010/2011 ${ }^{(1)}$.

\begin{tabular}{lcc}
\hline Tratamento & Antocianinas & $\begin{array}{c}\text { Compostos fenólicos } \\
\left(\mathrm{mg} \mathrm{g}^{-1}\right)\end{array}$ \\
\hline Fungicida & --------------- \\
Fertilizante & 3,5 & $17,9 \mathrm{a}$ \\
Phi A $\left(2,1 \mathrm{~g} \mathrm{~L}^{-1} \mathrm{P}_{2} \mathrm{O}_{5}\right)$ & 3,0 & $12,2 \mathrm{~b}$ \\
Phi B $\left(2,1 \mathrm{~g} \mathrm{~L}^{-1} \mathrm{P}_{2} \mathrm{O}_{5}\right)$ & 2,7 & $12,6 \mathrm{~b}$ \\
\hline
\end{tabular}

${ }^{(1)}$ Médias seguidas de letras iguais, nas colunas, não diferem pelo teste Scott-Knott, a $5 \%$ de probabilidade. Tratamento fungicida, aplicação alternada dos fungicidas Cabrio Top, Daconil e Ridomil. (2) Phi A, fosfito de potássio com formulação de $420 \mathrm{~g} \mathrm{~L}^{-1}$ de $\mathrm{P}_{2} \mathrm{O}_{5}$ e $280 \mathrm{~g} \mathrm{~L}^{-1}$ de $\mathrm{K}_{2} \mathrm{O}$; Phi $\mathrm{B}$, fosfito de potássio com formulação de $340 \mathrm{~g} \mathrm{~L}^{-1}$ de $\mathrm{P}_{2} \mathrm{O}_{5}$ e $240 \mathrm{~g} \mathrm{~L}^{-1}$ de $\mathrm{K}_{2} \mathrm{O}$; Fungicida, aplicação alternada de Cabrio Top, Daconil e Ridomil; Fertilizante, aplicação do fertilizante foliar Fulland. 
hipersensibilidade (morte celular), lignificação, ativação do metabolismo e aumento de fitoalexinas, além da atuação direta contra o patógeno (Daniel \& Guest, 2005). Apesar de ter sido verificada proteção contra o míldio da videira, pelas diferentes fontes de fosfito de potássio, observaram-se diferenças nos parâmetros avaliados que podem ser atribuídas aos tipos de componentes de cada formulação, aditivos e matéria prima de cada fabricante.

\section{Conclusões}

1. Os fosfitos de potássio são promissores no controle do míldio da videira e representam uma alternativa aos fungicidas utilizados na cultura.

2. A pulverização de fosfitos de potássio aumenta o teor de sólidos solúveis totais e o $\mathrm{pH}$, reduz a acidez total titulável das bagas e não afeta o teor de antocianinas.

\section{Agradecimentos}

Ao Conselho Nacional de Desenvolvimento Científico e Tecnológico, por concessão de bolsas; e à Empresa de Pesquisa Agropecuária de Minas Gerais, por suporte técnico.

\section{Referências}

ABE, L.T.; MOTA, R.V. da; LAJOLO, F.M.; GENOVESE, M.I. Compostos fenólicos e capacidade antioxidante de cultivares de uvas Vitis labrusca L. e Vitis vinifera L. Ciência e Tecnologia de Alimentos, v.27, p.394-400, 2007.

AMERINE, M.A.; OUGH, C.S. Methods for analysis of musts and wines. New York: John Wiley, 1980. 341p.

AZEVEDO, L.A.S. de. Manual de quantificação de doenças de plantas. São Paulo: Ciba Agro e Novartis, 1997. 114p.

BRUNETTO, G.; CERETTA, C.A.; KAMINSKI, J.; MELO, G.W.B. de; LOURENZI, C.R.; FURLANETTO, V.; MORAES, A. Aplicação de nitrogênio em videiras na Campanha Gaúcha: produtividade e características químicas do mosto da uva. Ciência Rural, v.37, p.389- 393, 2007.

CONDE, C.; SILVA, P.; FONTES, N.; DIAS, A.C.P.; TAVARES, R.M.; SOUSA, M.J.; AGASSE, A.; DELROT, S.; GERÓS, H. Biochemical changes throughout grape berry development and fruit and wine quality. Food, v.1, p.1-22, 2007.

CUŠ, F.; RASPOR, P. The effect of pyrimethanil on the growth of wine yeasts. Letters in Applied Microbiology, v.47, p.54-59, 2008.

DANIEL, R.; GUEST, D. Defence responses induced by potassium phosphonate in Phytophthora palmivora-challenged Arabidopsis thaliana. Physiological and Molecular Plant Pathology, v.67, p.194-201, 2005.

DUGO, G.; SAITTA, M.; GIUFFRIDA, D.; VILASI, F.; LA TORRE, G.L. Determination of resveratrol and other phenolic compounds in experimental wines from grapes subjected to different pesticide treatments. Italian Journal of Food Science, v.16, p.305-321, 2004.

ELRICH, K. Official methods of analysis of the Association of Official Analytical Chemists. $15^{\text {th }}$ ed. Arlington:: AOAC International, 1990. 2v.

GIL, G.F.; PSZCZÓLKOWSKI, P. Viticultura: fundamentos para optimizar producción y calidad. Santiago: Universidad Católica de Chile, 2007. 535p.

GIUSTI, M.M.; WROSLTAD, R.E. Characterization and measurement of anthocyanins by UV-visible spectroscopy. New York: John Willey, 2000. Unit F1.2. DOI: 10.1002/0471142913. faf0102s00. (Current protocols in food analytical chemistry).

GOMES, E.C.S.; LEITE, R.P.; SILVA, F.J.A.; CAVALCANTI, L.S.; NASCIMENTO, L.C.; SILVA, S.M. Manejo do míldio e ferrugem em videira com indutores de resistência: produtividade e qualidade pós-colheita. Tropical Plant Pathology, v.35, p.332-335, 2011.

GONZÁLEZ ÁLVAREZ, M.; NOGUEROL-PATO, R.; GONZÁLEZ-BARREIRO, C.; CANCHO GRANDE, B.; SIMAL-GÁNDARA, J. Changes of the sensorial attributes of white wines with the application of new anti-mildew fungicides under critical agricultural practices. Food Chemistry, v.130, p.139-146, 2012.

HOFMANN, U. Optimisation of downy mildew (Plasmopara viticola) control in organic viticulture with low copper doses, new copper formulations and plant strengtheners, results of 20 years of on farm research. Geisenheim: International Consultancy of Organic Viticulture, 2008. Available at: <http:// www.eco-consult.net>. Accessed on: 16 Aug. 2011.

HUKKANEM, A.T.; KOKKO, H.I.; BUCHALA, A.J.; MCDOUGALL, G.J.; STEWART, D.; KARENLAMPI, S.O. Benzothiadiazole induces the accumulation of phenolics and improves resistance to powdery mildew in strawberries. Journal of Agricultural and Food Chemistry, v.55, p.1862-1870, 2007.

IRITI, M.; VITALINI, S.; DI TOMMASO, G.; D’AMICO, S.; BORGO, M.; FAORO, F. New chitosan formulation prevents grapevine powdery mildew infection and improves polyphenol content and free radical scavenging activity of grape and wine. Australian Journal of Grape and Wine Research, v.17, p.263-269, 2011.

JACKSON, T.J.; BURGESS, T.; COLQUHOUN, I.; HARDY, G.E.S.T.J. Action of the fungicide phosphite on Eucalyptus marginata inoculated with Phytophthora cinnamomi. Plant Pathology, v.49, p.147-154, 2000.

LI, J.; ZINGEN-seLL, I.; BUCHENAUER, H. Induction of resistance of cotton plants to Verticillium wilt and of tomato plants to Fusarium wilt by 3-aminobutyric acid and methyl jasmonate. Journal of Plant Diseases and Plant Protection, v.103, p.288-299, 1996.

Pesq. agropec. bras., Brasília, v.47, n.11, p.1581-1588, nov. 2012 
MADDEN, L.V.; ELLIS, M.A.; LALANCETTE, N.; HUGHES, G.; WILSON, L.L. Evaluation of a disease warning system for downy mildew of grapes. Plant Disease, v.84, p.549-554, 2000.

MULLINS M.G.; BOUQUET, A.; WILLIAMS, L.E. Biology of the grapevine. Cambridge: Cambridge University, 1992. 239p.

MUÑOZ-ESPADA, A.C.; WOOD, K.V.; BORDELON, B.; WATKINS, B.A. Anthocyanin quantification and radical scavening capacity of Concord, Norton, and Marechal Foch grapes and wines. Journal of Agricultural and Food Chemistry, v.52, p.6779-6786, 2004.

NOJOSA, G.B.A.; RESENDE, M.L.V.; RESENDE, A.V. Uso de fosfitos e silicatos na indução de resistência. In: CAVALCANTE, L.S.; DI PIERO, R.M.; CIA, P.; PASCHOLATI, I.S.F.; RESENDE, M.L.V. de; ROMEIRO, R. da S. (Ed.). Indução de resistência em plantas a patógenos e insetos. Piracicaba: FEALQ, 2005. p.139-153.

PEREIRA, V.F.; RESENDE, M.L.V. de; MONTEIRO, A.C.A.; RIBEIRO JÚNIOR, P.M.; REGINA, M. de A.; MEDEIROS, F.C.L. Produtos alternativos na proteção da videira contra o míldio. Pesquisa Agropecuária Brasileira, v.45, p.25-31, 2010.

PERUCH, L.A.M.; BRUNA, E.D. Relação entre doses de calda bordalesa e de fosfito potássico na intensidade do míldio e na produtividade da videira cv. 'Goethe'. Ciência Rural, v.38, p.2413-2418, 2008.
SANTOS, H.A. dos; DALLA PRIA, M.; SILVA, O.C.; MAY DE MIO, L.L. Controle de doenças do trigo com fosfitos e acibenzolar-s-metil isoladamente ou associados a piraclostrobina + epoxiconazole. Semina: Ciências Agrárias, v.32, p.433-442, 2011.

SAUTTER, C.K.; STORCK, L.; RIZZATI, M.R.; MALLMANN, C.A.; BRACKMANN, A. Síntese de trans-resveratrol e controle de podridão em maçãs com uso de elicitores em pós-colheita. Pesquisa Agropecuária Brasileira, v.43, p.1097-1103, 2008.

SILVA, O.C.; SANTOS, H.A.A.; DALLA PRIA, M.; MAY-DE MIO, L.L. Potassium phosphite for control of downy mildew of soybean. Crop Protection, v.30, p.598-604, 2011.

SÔNEGO, O.R.; GARRIDO, L. da R. Avaliação da eficácia de algumas marcas comerciais de fosfito de potássio e de fosfonato de potássio no controle do míldio da videira. Bento Gonçalves: Embrapa Uva e Vinho, 2005. 13p. (Embrapa Uva e Vinho. Circular Técnica, 60).

SÔNEGO, O.R.; GARRIDO, L. da R.; GRIGOLETTI JÚNIOR, A. Principais doenças fúngicas da videira no Sul do Brasil. Bento Gonçalves: Embrapa Uva e Vinho, 2005. 25p. (Circular técnica, 56).

STADINIK, M.J.; TALAMINI, V. (Ed.). Manejo ecológico de doenças de plantas. Florianópolis: UFSC, 2004.

Recebido em 7 de maio de 2012 e aprovado em 15 de outubro de 2012 